Short communication

\title{
Colocalization of ribonuclear inclusions with muscle blind like-proteins in a family with myotonic dystrophy type 2 associated with a short CCTG expansion
}

\author{
S. Lucchiari a,*, S. Pagliarani a , S. Corti a , E. Mancinelli ${ }^{\text {b }}$, M. Servida ${ }^{\text {a }}$, E. Fruguglietti ${ }^{\text {a }}$, V. Sansone ${ }^{\text {c }}$, \\ M. Moggio ${ }^{a}$, N. Bresolin ${ }^{\text {a,d }}$, G.P. Comi ${ }^{\text {a }}$, G. Meola ${ }^{c}$

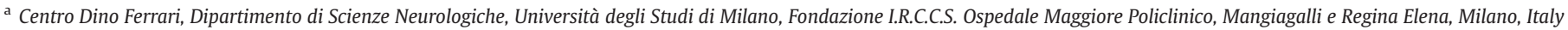 \\ b Dipartimento di Scienze Biomolecolari e Biotecnologie, Università degli Studi di Milano, Italy \\ c Unità Operativa di Neurologia-Stroke Unit, I.R.C.C.S. Policlinico San Donato, Università degli Studi di Milano, Italy \\ ${ }^{\mathrm{d}}$ I.R.C.C.S. Eugenio Medea, Bosisio Parini, Lecco, Italy
}

\section{A R T I C L E I N F O}

\section{Article history:}

Received 28 March 2008

Received in revised form 29 July 2008

Accepted 6 August 2008

Available online 18 September 2008

\section{Keywords:}

Myotonic dystrophy type 2

CCTG expansion

Muscle blind

\begin{abstract}
A B S T R A C T
Myotonic dystrophy type 2 (DM2) is an autosomal dominant multisystemic disorder caused by a CCTG repeat expansion in intron 1 of the zinc finger protein 9 (ZNF9) gene.

We present a three first-degree relative Italian family (proband, his mother and his sister) with a mild DM2 phenotype associated with a short (CCTG) $)_{100}$ expansion as far as regards the proband and his mother, while his sister shows larger expansion correlated to a more severe phenotype. FISH analysis with (CAGG) 5 probe demonstrated that nuclear foci of mutant RNA were present in the proband muscle and co-localized with muscleblind-like proteins, determining their sequestration in the nucleus. This is one of the smallest expansion reported and the shortest with the evidence of nuclear foci. These data contribute to the clinical and molecular correlation of ZNF9 gene short expansion.
\end{abstract}

(C) 2008 Elsevier B.V. All rights reserved.

\section{Introduction}

Myotonic dystrophies (DMs) are autosomal dominant multisystemic diseases that encompass two known genetic subtypes: myotonic dystrophy type 1 (DM1; OMIM \#160900) and type 2 which includes also as proximal myotonic myopathy (DM2/PROMM; OMIM \#602668). Clinical features include myotonia, muscular dystrophy, cardiac conduction defects, subcapsular cataracts and other systemic features such as hypogonadism, insulin resistance, and hypogammaglobulinemia [1]. However, most of DM2 patients appear to have a more favorable long-term prognosis as they do not show late deterioration in mental status, hypersomnia, dysphagia, gastrointestinal hypermotility, hearing deficits, ptosis, or other respiratory complications, features often present in cases of classic DM1. Cardiac arrhythmia is present in a minority of the patients. The severity of this disease is quite variable. No congenital case has ever been observed [2,3].

DM2 is caused by a CCTG expansion in intron 1 of ZNF9 gene (3q13.3q24, Locus ID\#AY329622) [4] coding for zinc finger protein-9 involved in a CAP-independent translation of cellular transcripts [5]. The pathologic

\footnotetext{
* Corresponding author. Sabrina Lucchiari, Centro Dino Ferrari, Dipartimento di Scienze Neurologiche, Università degli Studi di Milano, Fondazione I.R.C.C.S. Ospedale Maggiore Policlinico, Via Sforza 35, 20122 Milano, Italy. Tel.: +39 02 55033843; fax: +39 02503020430

E-mail address: sabrina.lucchiari@unimi.it (S. Lucchiari).
}

allele consists in an expansion of the complex repeat tract (TG) $)_{n}(\mathrm{TCTG})_{n}$ $(\mathrm{CCTG})_{n}$ that accumulates as ribonuclear inclusions and may alter activity of RNA-binding proteins such as muscle blind like-proteins (MBNL). In unaffected individuals the repeat is up to 27 CCTG-long, whereas in patients 75-11000 CCTG repeats were observed [3], and often more than one expanded allele can be detected in a single patient. No significant correlation between the age of onset and expansion size was observed. Still debated is the smallest pathogenic size of the expansion.

Here, we describe an Italian family with DM2 clinical, electrophysiological, and muscle biopsy features associated with a variable CCTG expansion. A very short (CCTG) 100 expansion was detected in two patients, the proband and his mother, determining a pathogenic effect by nuclear sequestration of MBNL1 (Muscle blind-like protein 1).

\section{Patients}

\subsection{Patient 1 (proband)}

The proband is a 32 year-old man presenting with the symptoms of "stiffness", myalgia and proximal leg weakness. Since his childhood, he began to notice difficulties in initiating his leg movements such as at the onset of physical exercise or in sprint running. This sensation would last few seconds, following which he could continue the motor activity without further difficulty. Over the years, this symptom demonstrated mild progression and did not vary with temperature or 
other environmental factors. After exercise, he complained of muscle fatigue. His past medical history was otherwise unremarkable and he took no medications.

On examination, cognitive function and cranial nerves were intact. He has moderate frontal balding and no temporal muscle atrophy. Handgrip myotonia was evident. His examination revealed no facial or neck flexor and extensor weakness, moderate shoulder girdle (Medical Research Council, MRC 4) and mild proximal (elbow flexors and extensors MRC 4) weakness, normal distal arm and hand strength (MRC 5). Examination of the lower extremities revealed mild pelvicgirdle (ileopsoas, MRC 4) and moderate distal leg muscle weakness (MRC 4). No muscle atrophy was noted. The remainder of the neurological examination was unremarkable.

Serum creatine kinase (CK) levels varied between 210 and 320 U/L (n.v. $180 \mathrm{U} / \mathrm{L}$ ). Other blood examinations, including thyroids stimulating hormone levels, were normal.

Electromyography (EMG) showed widespread myotonic discharges, as well as occasional positive sharp waves in a number of regions including the right deltoid, supraspinatus, and biceps. In these muscles, motor unit potentials were mildly reduced in amplitude and duration, whereas recruitment and firing rate were normal. Nerve conduction studies were normal.

Brain MRI, split-lamp examination, electrocardiography, echocardiography and respiratory function were normal.

\subsection{Patient 2}

The mother of patient 1 was evaluated at age 58 years. She noted in her forties muscle fatigue after physical exercise. She complained also of myalgias. She denied muscle stiffness or other muscle symptoms.

On examination, cognitive function and cranial nerves were intact. There were no clinical signs of myotonia. The strength of facial, neck flexor and extensor muscles was normal. Shoulder girdle, elbow flexors and extensors were moderately weak (MRC 4). Distal arm and hand strength was normal (MRC 5). Examination of the lower extremities revealed mild pelvic-girdle weakness (MRC 4) and normal distal leg strength. No muscle atrophy was noted.

Split-lamp examination, electrocardiography and respiratory function were normal. Echocardiography showed an ejection fraction of $65 \%$, the other parameters were normal. CK levels were between 224 and $250 \mathrm{U} / \mathrm{L}$. Nerve conduction studies and EMG were normal.

\subsection{Patient 3}

The older sister of the proband, a 35 year-old woman, showed a more clinical severe DM2 phenotype compared with her relatives. Her symptoms started at around the age of 5 years, when she presented mild leg weakness and myalgia. Later during her childhood she complained of generalized limb weakness and significant muscle stiffness, usually at the onset of physical activity. At school, she was unable to practice sports and she noticed the improvement of her stiffness with activity.

She had no abortions and did not complain about worsening of symptoms, e.g. stiffness, myotonia or weakness, during the course of pregnancies or delivery of her two children that were referred as healthy. Her past medical history was otherwise unremarkable and she took no medications.

At the neurological examination, she has grip myotonia, weakness of the neck flexors (sternocleidomastoideus, MRC 4) moderate shoulder girdle weakness (MRC 4), and mild proximal and distal arm and hand weakness (finger flexors, MRC 4). In the lower limbs, weakness was symmetrical and mostly proximal, with weak muscles graded as follows: 4 hip flexors and extensors, 4 hip abductors, 4 knee flexors and extensors, 5 ankle dorsiflexion, all symmetrical. She could not get up from the ground in a squatting position. She was able to walk unaided, but had difficulty rising from a chair and sitting up from supine.

No muscle atrophy was evident. She had neither signs of mental retardation nor cognitive impairment. The remainder of the neurological examination was unremarkable.

No cataract was present. Her electrocardiographic and echocardiographic examinations were normal. Nerve conduction velocities were normal. EMG showed myotonic runs in limb muscles. In the laboratory examination, complete blood count and blood biochemistry were normal. CK ranged between 247 and $349 \mathrm{U} / \mathrm{l}$.

\section{Methods}

\subsection{Histopathological studies}

After informed consent, a muscle biopsy was taken from the left biceps from patient 1 at the age of 32 years, according to a protocol approved by the Institutional Review Board of the "IRCCS Foundation Ospedale Maggiore Policlinico, Mangiagalli e Regina Elena, Milan, Italy”. Muscle tissue was fresh-frozen in liquid nitrogen-cooled isopentane, sectioned at $8 \mu \mathrm{m}$. Cryostatic cross sections were processed according to standard histological/histochemical techniques [6].

\subsection{Genetic analysis}

Blood samples were obtained from the three patients, after informed consent. In order to preserve the integrity of genomic DNA samples, extraction from peripheral leukocytes was performed following the "salting out" protocol. DNA was extracted as well from cryopreserved muscle biopsy of patient 1 . Genetic screening for DM1 (DMPK gene) was first performed and the outcome was negative. Subsequently, we tested the patients for DM2. As first step, a PCR-based allele sizing across marker CL3N58, which includes the CCTG repeat stretch of the ZNF9 gene, was made in order to check for normal alleles using primers previously described [4], so that the wild-type PCR products range from $250 \mathrm{bp}$ to $280 \mathrm{bp}$. Briefly, the amplification was performed in 35 cycles,
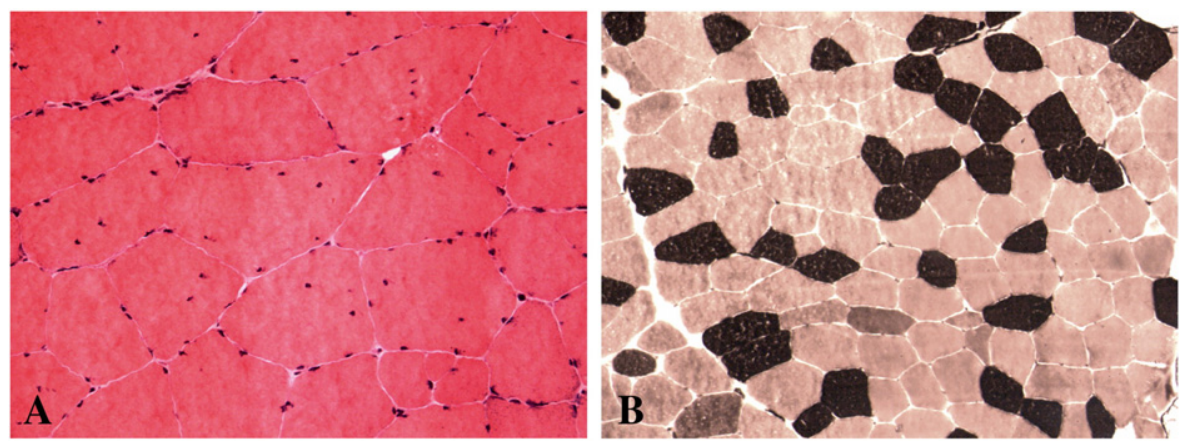

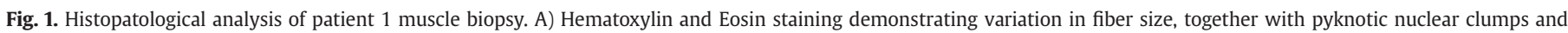
scattered angulated atrophic fibers (250×). B) ATPase $\mathrm{pH} 4.6$ evidencing small type II grouping fibers (100×). 
with a $T_{\mathrm{a}}$ at $56{ }^{\circ} \mathrm{C}$, elongation at $72{ }^{\circ} \mathrm{C}$ for $30 ", 30 \mu \mathrm{M}$ each primer using GoTaq DNA Polymerase by Promega (Promega, WI, USA, www.promega. com). Afterwards, DNA both from blood and from muscle went through a XL-PCR to draw out possible expanded alleles. Briefly, 100 and $200 \mathrm{ng}$ of DNA were amplified using the same primer pair and TaKaRa LATaq 1.5 U (TAKARA BIO INC., Japan, www.takara-bio.co.jp), with a $10 \mathrm{~min}$ extension step. Amplified DNA was run on a $1 \%$ agarose gel, blotted onto a nylon membrane, and hybridized to a ${ }^{32}$ P-labelled probe.

Amplification products were cloned to seize their expansions in TOPO-TA Cloning system (Invitrogen, Carlsbad, CA, USA, www. invitrogen.com) and sequenced by Big Dye Terminator Cycle Sequencing Kit in an automated sequencer 3100 (Applied Biosystem, Foster City, USA, www.appliedbiosystems.com).

\subsection{Fluorescent in situ hybridization analysis (FISH) and Muscleblind 1 (MBNL1) immunofluorescence}

FISH was performed on frozen muscle sections from patient 1 using a (CAGG) $)_{5}$ probe as previously reported [7].

Following the post-hybridization wash, the immunofluorescence protocol for MBLN1 was performed as previously described [8], using a polyclonal rabbit anti-MBNL1 (gift of Prof. C.A. Thornton, University of Rochester, New York, USA).

The interaction between ribonuclear inclusions and MBNL1 nuclear accumulation was examined by analysis of images obtained at confocal microscopy.

\section{Results}

\subsection{Histopathological studies}

Histology performed on the proband's muscle showed a myopathic pattern with an increased centrally nucleated myofibers having pronounced fibre size variability, nuclear clumps and a preferential atrophy of type 2 fibres. A mild increase of endomysial connective tissue was present. Necrosis or inflammatory infiltrate was not detectable (Fig. 1).

\subsection{Genetic analysis}

Amplification of CL3N58 marker detected a single normal allele of $252 \mathrm{bp}$, presenting an interrupted expansion of 17 CCTG in the proband and in patient 3, and $260 \mathrm{bp}$ in patient 2, with a non continuous (CCTG) ${ }_{19}$. Southern blot revealed the presence of one band whose size is comprised between 500 and $600 \mathrm{bp}$, and corresponds to an expansion of about 100 CCTG in patients 1 (blood and muscle) and 2 (blood) (Fig. 2).

Analysis by cloning further demonstrated in both patients an uninterrupted (CCTG) 32 -repeat pre-expansion. The wide lower spots present in both proband and patient 2 is likely to include the preexpansion alleles. Southern blot analysis on patient 3 showed a large expanded allele of approximately $2.5 \mathrm{~kb}$. Each patient was proven negative for mutations in CLCN1 gene coding for skeletal muscle chloride channel, known to possibly worsen the DM2 clinical phenotype [9].

\subsection{FISH analysis}

The ribonuclear inclusions formed by pathologic retained expansions in DM2 can be visualized by fluorescent in situ hybridization (FISH). Immunofluorescence analysis in human skeletal muscle section from patient 1 showed that ribonuclear inclusions co-localize with MBNL1 foci within muscle nuclei (Fig. 3). The confocal microscopy images confirmed the co-localization between ribonuclear inclusions (red) and MBNL1 foci (green), both merged (yellow) in nucleus (blue) (Fig. 3). We detected the

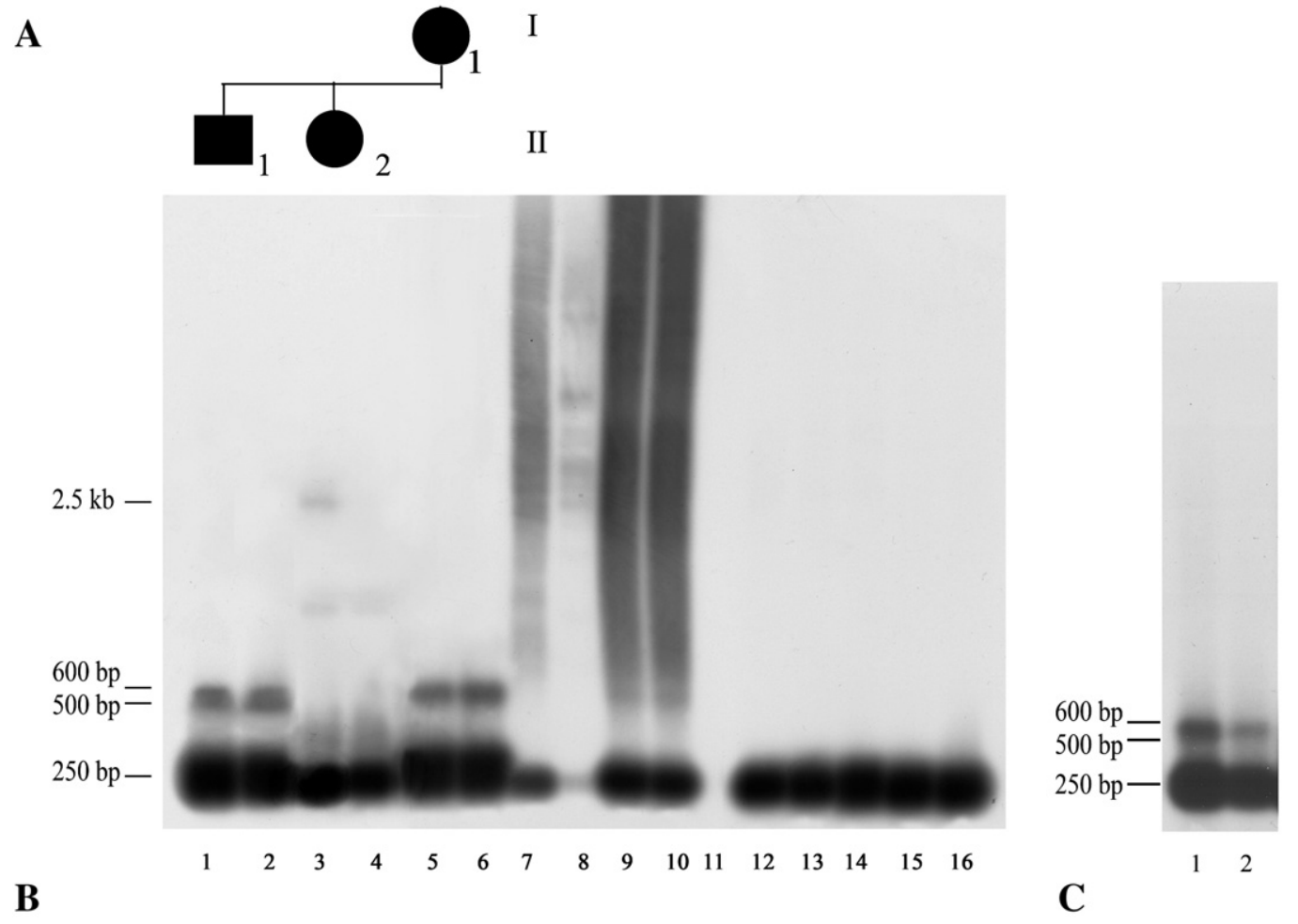

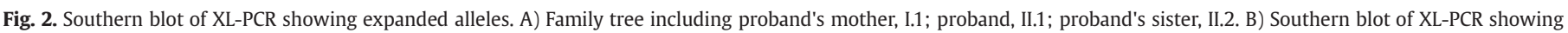

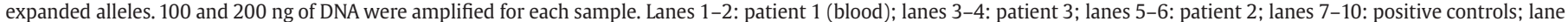

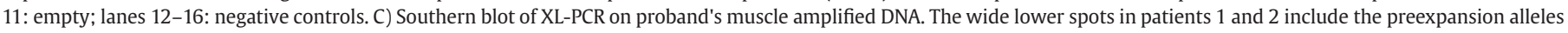
$(\mathrm{CCTG})_{32}$, whereas the bands comprised between 500 and 600 bp correspond to up (CCTG) 100 repeats. 

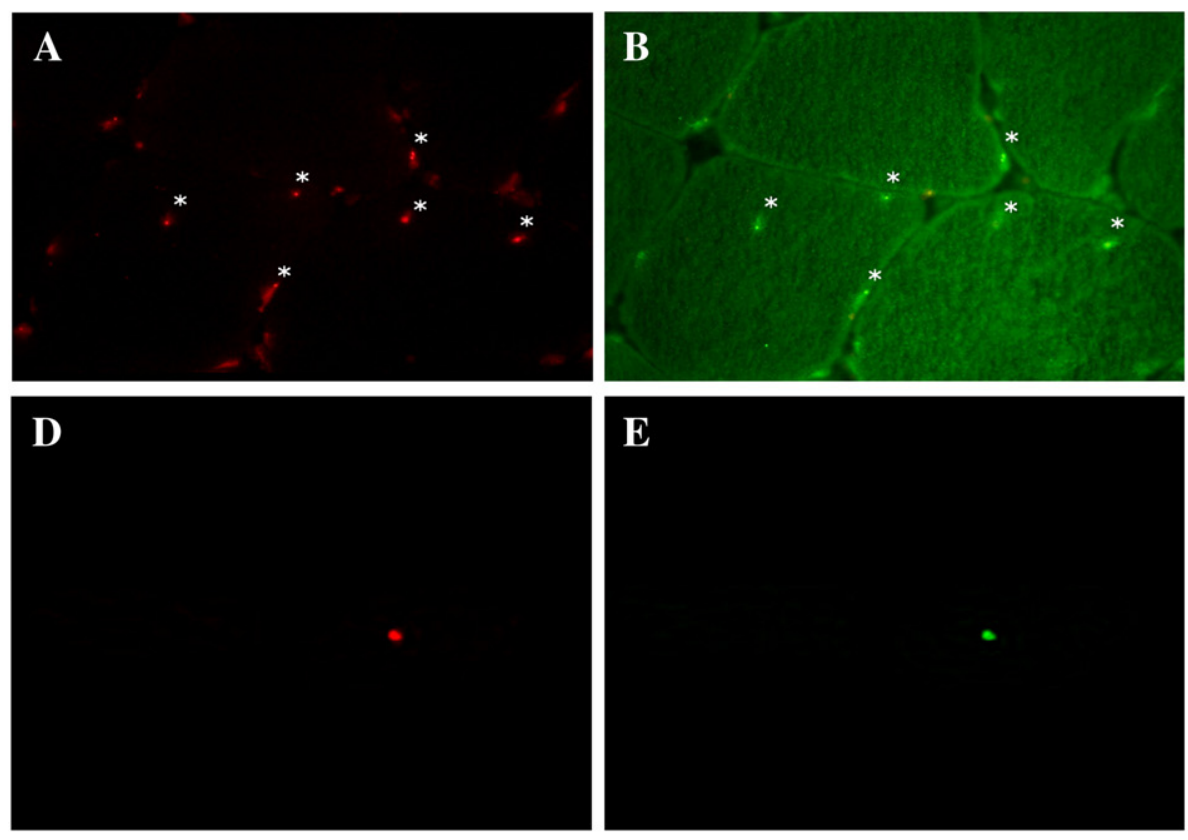
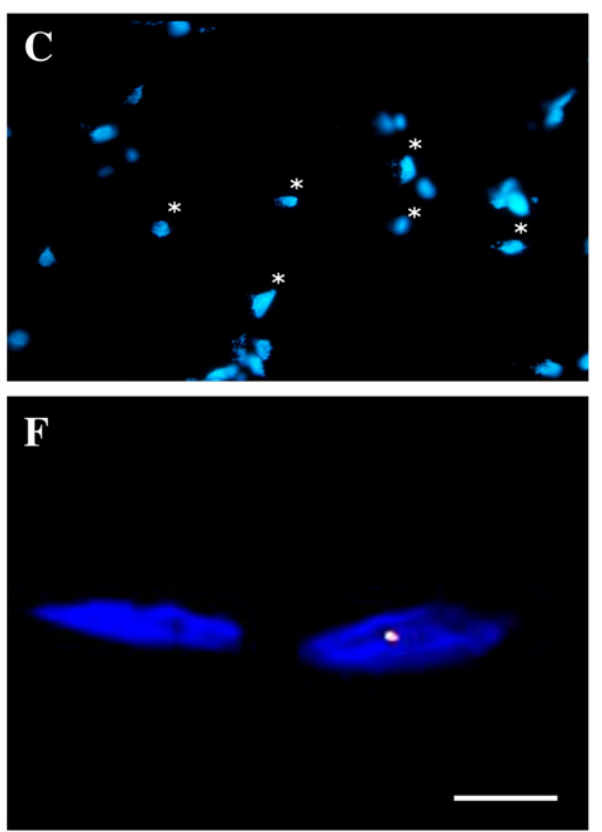

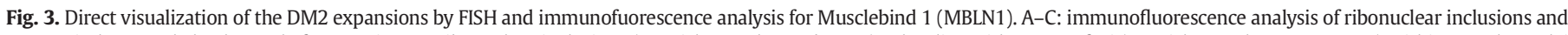

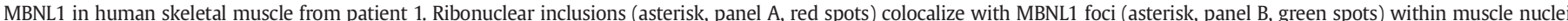

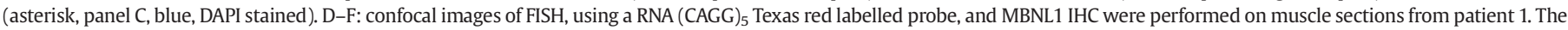
ribonuclear inclusions (panel A, red spot) and MBNL1 foci (panel B, green spot) merge (panel C, yellow spot) in nucleus (panel C, blue). Scale Bar: A-C: 100 um; D-F: $4 \mu \mathrm{m}$.

expression of nuclear foci and MBLN1 in over $70 \%$ of nuclei with a rate of colocalization $>90 \%$.

\section{Discussion}

In DM1, there is a direct correlation between expansion size and clinical severity of the disease, the expansion tends to increase when transmitted from one generation to the next and pathologic alleles are referred to fixed ranges of expansion [10].

In DM2, although anticipation has been reported, there is no obvious evidence for intergenerational expansions. In addition, there is no clear correlation between the size of the (CCTG)n expansion and the severity of the clinical phenotype [3]. Furthermore, there is the need to discriminate pre-mutational alleles from small pathogenic alleles.

As described by Liquori et al. [11], alleles displaying an uninterrupted stretch of 20 CCTG can be valued as premutational. Indeed, the repeat that was found in patients 1 and 2 by cloning comprised 32 succeeding CCTG without interruption. They showed as well a larger expansion. As in none of our patients we found the coexistence of wild-type, premutational and expanded alleles it would have been of interest to analyze previous generations from this family to check the stability of the premutational expansion.

Furthermore, we detected an expansion of 100 CCTG in blood of patient 1 and 2 and in the muscle of patient 1 . This is one of the shortest expansions reported in the literature. It has been previously mentioned in one large collection of DM2 patient, the detection of a 75 CCTG expansion, but without a detailed clinical report [3].

Our three first-degree relatives demonstrated a different combination of clinical, biochemical and electrophysiological features with two of them carrying the same CCTG expansion. Clinical features of the patients described in this study are typical of DM 2 [12-14].

Patient 1, with a short CCTG expansion, presented symptoms of muscle stiffness beginning in his childhood. He showed chronic myopathic changes in his muscle biopsy and FISH analysis by (CAGG) probe demonstrated that nuclear foci of mutant RNA were present in his muscle and colocalize with MBNL1, further confirming the diagnosis. Patient 1 pattern of expansion in muscle was like his one in blood (Fig. 2).
On the other hand, patient 3 carrying a larger expansion presented myotonia and weakness since her childhood, with a degree of disability slightly severe than his brother, likely correlating her worse phenotype with molecular findings.

In the muscle biopsy of patient 1 with short expansion, we were able to detect the presence of nuclear foci and their co-localization with MBLN1. This is the shortest expansion in which this analysis was performed. Our finding establishes the lowest bounds of sensitivity of the described protocol.

These data suggest that expansion of 100 CCTG are long enough to be retained in the nuclei. This is in contrast with that observed in DM1 where an incomplete nuclear retention of short repeats from minimally affected patients is found. In these cases, small (150 CUG) repeats were associated to nuclear foci, but only in approximately $15 \%$ of nuclei [15].

Current models of DM1 and DM2 suggest a "spliceopathy" as the primary pathogenic mechanism and, as a consequence, the failure of the essential switch from an embryonic to adult splicing pattern. This results in aberrant expression of embryonic isoforms that are unable to support the functional requirements of adult tissues [16].

The effects of "spliceopathy" on different transcripts may provide a basis for the multisystemic features of DM1 and DM2.

In conclusion, the findings confirm the broad spectrum of clinical, electrophysiological and pathological manifestations of DM2 patients that can be seen within the same family. These data contribute to the definition of the minimal pathogenetic size of CCTG expansion allowing the recognition and diagnosis of such disease.

\section{Acknowledgments}

We wish to thank especially the 'Associazione Amici del Centro Dino Ferrari' for their support. The Telethon Genetic Biobanks Network GTB07001E and the Eurobiobank project are gratefully acknowledged.

The financial support of the following research grants is gratefully acknowledged: MIUR (Ministero Istruzione Università di Ricerca Scientifica) Italian Ministery PRIN 2007, PRIN MIUR 2006 to Giovanni Meola. Co-author S. Pagliarani is supported by a fellowship of the 
Doctorate School of Molecular Medicine, University of Milan. A lot of gratitude has to be express to the patients for participating in research and for their kindly collaboration.

\section{References}

[1] Wheeler TM, Thornton CA. Myotonic dystrophy: RNA-mediated muscle disease. Curr Opin Neurol 2007;20(5):572-6.

[2] Schara U, Schoser BG. Myotonic dystrophies type 1 and 2: a summary on current aspects. Semin Pediatr Neurol 2006;13(2):71-9.

[3] Day JW, Ricker K, Jacobsen JF, Rassmussen LJ, Dick KA, Kress W, et al. Myotonic dystrophy type 2: molecular, diagnostic and clinical spectrum. Neurology 2003;60 (4):657-64.

[4] Liquori CL, Ricker K, Moseley ML, Jacobsen JF, Kress W, Naylor SL, et al. Myotonic dystrophy type 2 caused by a CCTG expansion in intron 1 of ZNF9. Science 2001;293(5531):864-7.

[5] Gerbasi VR, Link AJ. The myotonic dystrophy type 2 protein ZNF9 is part of an ITAF complex that promotes cap-independent translation. Mol Cell Proteomics 2007;6(6):1049-58.

[6] V. Dubowitz, Muscle biopsy: A practical approach. Balliére-Tindall W. B. Saunders, London, Toronto 1985.

[7] Cardani R, Mancinelli E, Sansone V, Rotondo G, Meola G. Biomolecular identification of (CCTG)n mutation in myotonic dystrophy type 2 (DM2) by FISH on muscle biopsy. Eur J Histochem 2004;48:437-42.
[8] Cardani R, Mancinelli E, Rotondo G, Sansone V, Meola G. Muscleblind-.like protein 1 nuclear sequestration is a molecular pathology marker of DM1 and DM2. Eur J Histochem 2006;50(3):177-82.

[9] Suominen T, Schoser B, Raheem O, Auvinen S, Walter M, Krahe R, et al. Report of the 6th International Myotonic Dystrophy Consortium Meeting workshop, 12-15 September 2007, Milan, Italy, vol. 046; 2007. p. 15.

[10] Harper PS. Myotonic dystrophy. 3rd ed. London: W. B. Saunders; 2001.

[11] Liquori CL, Ikeba Y, Weatherspoon M, Ricker K, Schoser BG, Dalton JC, et al. Myotonic dystrophy type 2: human founder haplotype and evolutionary conservation of the repeat tract. Am J Hum Genet 2003;73(4):849-62.

[12] Meola G, Moxley RT. Myotonic dystrophy type 2 and related myotonic disorders. J Neurol 2004;251:1173-82.

[13] Moxley RT, Meola G. The myotonic dystrophies. In: Rosenberg RN, Di Mauro S, Paulson HL, Ptacek L, Nestler EJ, editors. The molecular and genetic basis of neurologic and psychiatric disease. 4th Ed. Philadelphia, Lippincot Williams; 2008. Chapter 47.

[14] Botta A, Bonifazi E, Vallo L, Gennarelli M, Garrè C, Salehi L, et al. Italian guidelines for molecular analysis in myotonic dystrophies. Acta Myol 2006;25(1):23-33

[15] Davis BM, McCurrach ME, Taneja KL, Singer RH, Housman DE. Expansion of a CUG trinucleotide repeat in the 3' untranslated region of myotonic dystrophy protein kinase transcripts results in nuclear retention of transcripts. Proc Natl Acad Sci USA 1997;8;94(14):7388-93.

[16] Osborne RJ, Thornton CA. RNA dominant diseases. Hum Mol Genet 2006;15: R162-9 (special n. 2). 